\title{
EDITORIAL
}

\section{Novel membrane separation technologies and membrane processes}

\author{
Yanying Wei $(\bowtie)^{1}$, Gongping Liu $(\bowtie)^{2}$, Jianquan Luo $(\bowtie)^{3}$, Libo Li $(\bowtie)^{1}$, Zhi Xu $(\bowtie)^{4}$ \\ 1 School of Chemistry \& Chemical Engineering, South China University of Technology, Guangzhou 510640, China \\ 2 State Key Laboratory of Materials-Oriented Chemical Engineering, College of Chemical Engineering, Nanjing Tech University, \\ Nanjing 211816, China \\ 3 State Key Laboratory of Biochemical Engineering, Institute of Process Engineering, University of Chinese Academy of Sciences, \\ Chinese Academy of Sciences, Beijing 100190, China \\ 4 State Key Laboratory of Chemical Engineering, School of Chemical Engineering, East China University of Science and \\ Technology, Shanghai 200237, China
}

(C) Higher Education Press 2021

Membrane-based separation technologies have received increasing attention attributing to lots of advantages such as the low energy consumption, easy operation, and environmental friendliness. In recent years, the emergence of novel two-dimensional (2D) materials, such as graphene, provides a new opportunity for membrane development. Thus, Frontiers of Chemical Science and Engineering (FCSE), one of the transactions of Chinese Academy of Engineering, organized this Special Issue aiming to illustrate some of the exciting progresses and achievements in the relevant research fields. In this Special Issue, we included seven review articles and six featured research papers from research groups from China and overseas, including 2D nanosheets membranes separation (MXene membranes, graphene oxide (GO) membranes, $\mathrm{MoS}_{2}$ membranes) and imprinted membranes, as well as several membrane processes, such as nanofiltration process, membrane distillation, membrane-coupled photodegradation, films for X-ray shielding and membrane contactors for $\mathrm{CO}_{2}$ capture.

This review titled "Recent progress of two-dimensional nanosheet membranes and composite membranes for separation applications", presents the development, progress, and recent break-through of different types of 2D membranes, including membranes based on porous and non-porous 2D nanosheets for various separations. And the separation mechanism of 2D membranes, their fabrication methods, challenges and future directions of 2D membranes for wide utilization are discussed in brief [1].

Emerging MXene-based membranes play significant roles in the competitive membrane-separation field. The review paper "Recent progress in the design and fabrication of MXene-based membranes", gives recent major advances of MXene-based membranes based on gas-molecule sieving, ion sieving, and other small-molecule sieving. New horizons for MXene-based membranes are highlighted from four aspects, including MXene nanosheet cross-linking, constructing additional nanochannels, MXene hybrid membranes, and MXenes as 2D scaffolds in this review [2].

As an alternative option, GO membranes show great potential in water-ethanol separations for producing bioethanol. "Theoretical study on Janus graphene oxide membrane for water transport" computationally design the Janus GO membranes for water separation from ethanol. They report that the Janus GO membrane with an OPOP orientation exhibited an exceptionally high water flux $\left(\sim 40.9 \mathrm{~kg} \cdot \mathrm{m}^{-2} \cdot \mathrm{h}^{-1}\right)$, highlighting the important roles of the oxidized upper surface and interlayer channel in water crossing through the aligned GO pores for selective water transport [3].

Moreover, the emerging 2D material nanopores have provided a new avenue for high performance DNA

Received January 5, 2021

E-mails: ceyywei@scut.edu.cn (Wei Y), gpliu@njtech.edu.cn (Liu G), jqluo@ipe.ac.cn (Luo J), celbli@scut.edu.cn (Li L), zhixu@ecust.edu.cn (Xu Z) 
sequencing, which is critical for modern chemical/biological research or applications. In "Molecular dynamics simulation on DNA translocating through $\mathrm{MoS}_{2}$ nanopores with various structures", Li et al. simulated DNAs translocating through various $\mathrm{MoS}_{2}$ nanopores in a stepwise manner, yielding stable signals (e.g., ionic current) for DNA sequencing. Various factors regulating the translocating process and associating ionic current were studied, providing with useful insights or guidelines for the development of nanopore-based DNA sequencing technologies [4].

Besides the separation applications of 2D membranes, some other membrane processes are also reviewed. Obtainment of sugar from biomass is important for food and biomanufacturing industries, and nanofiltration membranes with $0.5-2 \mathrm{~nm}$ pore size enable their use in fractionation and purification of sugar from biomass. The review paper "Nanofiltration for separation and purification of saccharides from biomass" summarizes recent advances on the applications of nanofiltration in separation of saccharides, provides an insight into the mechanisms of mass transfer and membrane fouling as well as membrane cleaning strategies, and discusses the challenges and possible solutions of membrane technology in sugar industry [5].

We sincerely thank the Editors-in-Chief and editorial team to assemble this Special Issue and hope the readers enjoy the articles.

\section{References}

1. Wang W, Wei Y, Fan J, Cai J, Lu Z, Ding L, Wang H. Recent progress of two-dimensional nanosheet membranes and composite membranes for separation applications. Frontiers of Chemical Science and Engineering, 2021, 15(4): 793-819

2. Qu K, Huang K, Xu Z. Recent progress in the design and fabrication of MXene-based membranes. Frontiers of Chemical Science and Engineering, 2021, 15(4): 820-836

3. Liu Q, Chen M, Mao Y, Liu G. Theoretical study on Janus graphene oxide membrane for water transport. Frontiers of Chemical Science and Engineering, 2021, 15(4): 913-921

4. Zhao D, Chen H, Wang Y, Li B, Duan C, Li Z, Li L. Molecular dynamics simulation on DNA translocating through MoS 2 nanopores with various structures. Frontiers of Chemical Science and Engineering, 2021, 15(4): 922-934

5. Li X, Tan S, Luo J, Pinelo M. Nanofiltration for separation and purification of saccharides from biomass. Frontiers of Chemical Science and Engineering, 2021, 15(4): 837-853

\section{Guest Editors}

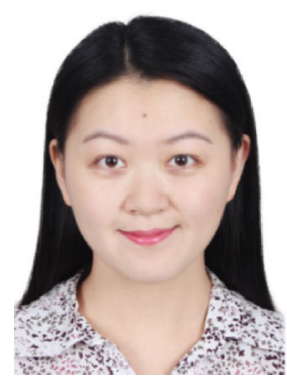

Prof. Yanying Wei received her Ph.D. degree under the supervision of Prof. Haihui Wang at South China University of Technology in 2013. During 2013-2015, she worked as a Humboldt research fellow in Prof. Juergen Caro's group at Leibniz University of Hannover. She is currently a professor in the School of Chemistry \& Chemical Engineering at South China University of Technology. Her research focuses on novel 2D nanosheets membranes, MOF membranes for various separation and catalytic membrane reactors. She has published over 70 peer-reviewed journal papers at Nat. Sustain., Nat. Commun., Sci. Adv., JACS, Angew. Chem. Int. Ed., etc., 2 co-authored monographs and held 39 patents.

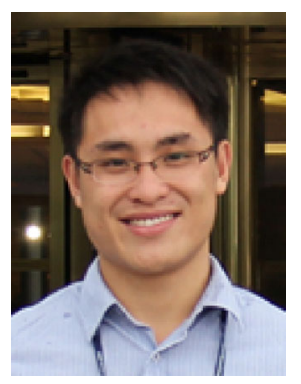

Prof. Gongping Liu received his Ph.D. degree under the supervision of Prof. Wanqin Jin at Nanjing University of Technology in 2013, and then joined Nanjing Tech University as an assistant professor. During 2015-2017, he worked as a postdoctoral fellow in Professor William J. Koros group at Georgia Institute of Technology. He is currently a professor in the College of Chemical Engineering at Nanjing Tech University. His research focuses on advanced membranes for molecular separations based on mixed-matrix and 2D materials. 

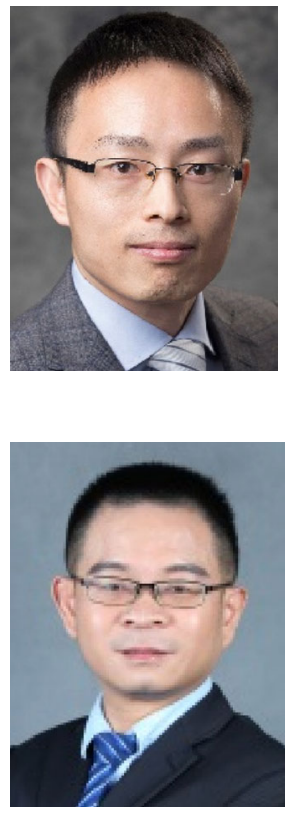

Prof. Libo Li received his B.S. (2001) and M.S. (2005) degrees from Tsinghua University, and Ph.D. (2011) from University of California at Davis (Advisor: Prof. Toby Allen). After working with Prof. Ken Dill at Stonybrook University as a postdoc (2011-2014), he joined South China University of Technology (China) as an Associate Professor, and is currently Full Professor. His research interests focus on simulating ion/molecule transportation in nanochannels, with implications for separation, sensing and energy storage/conversion. He has published over 70 peer-reviewed journal papers at Sci. Adv., Angew. Chem. Int. Ed., etc.

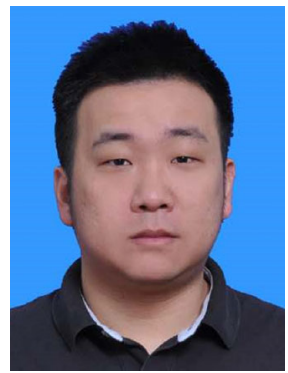

Prof. Zhi Xu joined East China University of Science and Technology (ECUST) in 2019 as a full professor. He obtained his Ph.D. degree from University of Cincinnati in 2015. Prior to joining ECUST, he has joined University of Cincinnati and University of Oxford as a postdoctoral fellow from 2015 to 2019. His current research interests focus on the synthesis of 2D materials and membrane separation. 